\title{
Large-grain MBE-grown GaSe on GaAs with a Mexican hat- like valence band dispersion
}

\author{
Ming-Wei Chen ${ }^{1,2}$, HoKwon Kim (1) ${ }^{1}$, Dmitry Ovchinnikov ${ }^{1}$, Agnieszka Kuc ${ }^{3}$, Thomas Heine $\mathbb{C}^{3}$, Olivier Renault ${ }^{4,5}$ and Andras Kis ${ }^{1,2}$
}

Atomically thin GaSe has been predicted to have a non-parabolic, Mexican hat-like valence band structure due to the shift of the valence band maximum (VBM) near the $\Gamma$ point which is expected to give rise to novel, unique properties such as tunable magnetism, high effective mass suppressing direct tunneling in scaled transistors, and an improved thermoelectric figure of merit. However, the synthesis of atomically thin GaSe remains challenging. Here, we report on the growth of atomically thin GaSe by molecular beam epitaxy (MBE) and demonstrate the high quality of the resulting van der Waals epitaxial films. The full valence band structure of nominal bilayer GaSe is revealed by photoemission electron momentum microscopy ( $k$-PEEM), confirming the presence of a distorted valence band near the $\Gamma$ point. Our results open the way to demonstrating interesting new physical phenomena based on MBE-grown GaSe films and atomically thin monochalcogenides in general.

npj 2D Materials and Applications (2018)2:2 ; doi:10.1038/s41699-017-0047-x

\section{INTRODUCTION}

Atomically thin two-dimensional (2D) materials have recently attracted great interest due to distinct electrical and optical properties, different from those of their bulk counterparts. ${ }^{1-3}$ Gallium selenide (GaSe) belongs to the semiconducting 2D family and has a layered structure exhibiting in-plane covalent bonding and weak van der Waals interlayer interaction. In the unit cell, two $\mathrm{Ga}$ atoms are sandwiched by two planes of Se atoms, forming a monolayer with Se-Ga-Ga-Se stacking, Fig. 1a. Similarly to other 2D materials, the in-plane geometry has a honeycomb-like hexagonal structure. Bulk GaSe has a direct band-gap of $\sim 2.1 \mathrm{eV}$ and has been widely investigated for its superior second harmonic generation and photoresponse properties. ${ }^{4,5}$ In the monolayer limit, GaSe exhibits a direct-to-indirect band gap transition, and therefore shows an opposite trend to the thickness dependence in most transition metal dichalcogenides. ${ }^{6,7}$ As the number of layers is decreased below seven layers, the shape of the valence band close to its maximum at the $\Gamma$ point undergoes a change and develops a Mexican hat or caldera-like inverted plateau ${ }^{6,8,9}$ as shown in Fig. $1 b$.

This dispersion should give rise to a sharp van Hove singularity in the density of states resulting in the possibility of a ferromagnetic phase transition with hole doping in the monolayer limit. ${ }^{7,10}$ The high density of states at the valence band edge could also indicate the presence of a high thermoelectric power factor in this material ${ }^{11}$ while the flat valence band would imply a high hole effective mass, resulting in the suppression of direct tunneling in scaled transistors. ${ }^{12}$

Although the synthesis of GaSe by molecular beam epitaxy (MBE) can be traced back to early $1990^{\prime} \mathrm{s}^{13-16}$ the atomically thin nature of GaSe has been much less studied, and the studies of electronic properties were mostly based on the bulk material. ${ }^{17}$ Here, we address the main difficulties related to the growth of atomically thin and high-quality GaSe. First is the random orientation of as-grown films, which can take place during the growth. Although $\mu \mathrm{m}$-sized flakes have been synthesized by chemical vapor deposition (CVD), further investigation of the films using transmission electron microscopy (TEM) shows that resulting films have random grain orientations. ${ }^{8}$ The film morphology and uniformity are also difficult to control. Spiral and island growth modes were commonly reported for epitaxial GaSe films grown by $M B E,{ }^{15,18-20}$ making it more difficult to control the film thickness, especially in the atomically thin limit. While oscillations in reflection high energy electron diffraction (RHEED) patterns during the growth of GaSe on mica were used to determine the thickness, indicating that layer-by-layer growth could be achieved, ${ }^{21}$ the morphology evolution, particularly in atomically thin layers was not addressed at all. Device characterization is also still based on bulk GaSe. Overall, it is still challenging to develop a reliable way for synthesizing atomically thin GaSe films for further characterization with the aim of understanding the peculiar electronic properties of this material.

Here, we have used MBE with a two-step growth approach to synthesize high-quality GaSe with a $\mu \mathrm{m}$-scale grain size as seen from the uniformity of the work function and angular dependence of the valence band structure. As-grown films can be delaminated from the growth substrate and used for further studies, for example in scanning transmission electron microscopy (STEM). We have further investigated the electronic properties of nominal bilayer GaSe films by photoemission electron momentum microscopy ( $k$-PEEM) and confirmed the Mexican-hat shaped dispersion in the upper valence band. ${ }^{20}$ These results demonstrate that MBEgrown GaSe films can be an ideal platform for investigation of new physics in atomically thin monochalcogenides.

\footnotetext{
'Electrical Engineering Institute, École Polytechnique Fédérale de Lausanne (EPFL), 1015 Lausanne, Switzerland; ${ }^{2}$ Institute of Materials Science and Engineering, École Polytechnique Fédérale de Lausanne (EPFL), 1015 Lausanne, Switzerland; ${ }^{3}$ University of Leipzig, 04103 Leipzig, Germany; ${ }^{4}$ Université Grenoble Alpes, Grenoble 38000 , France and ${ }^{5}$ CEA, LETI, MINATEC Campus, Grenoble 38054, France

Correspondence: Andras Kis (andras.kis@epfl.ch)
}

Received: 20 October 2017 Revised: 20 December 2017 Accepted: 29 December 2017

Published online: 25 January 2018 

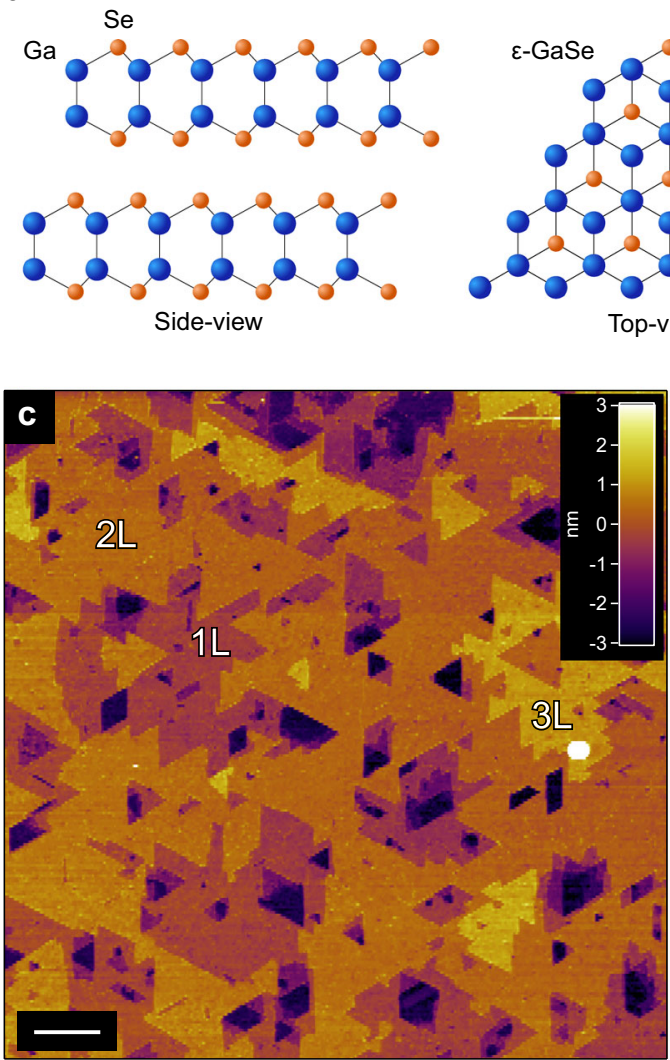

b
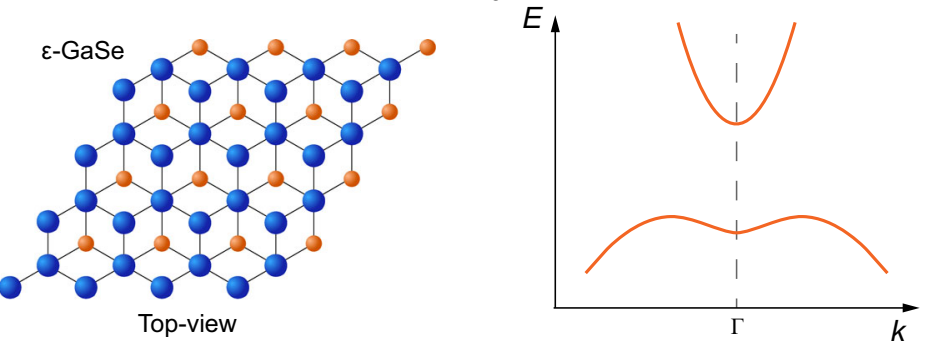

d

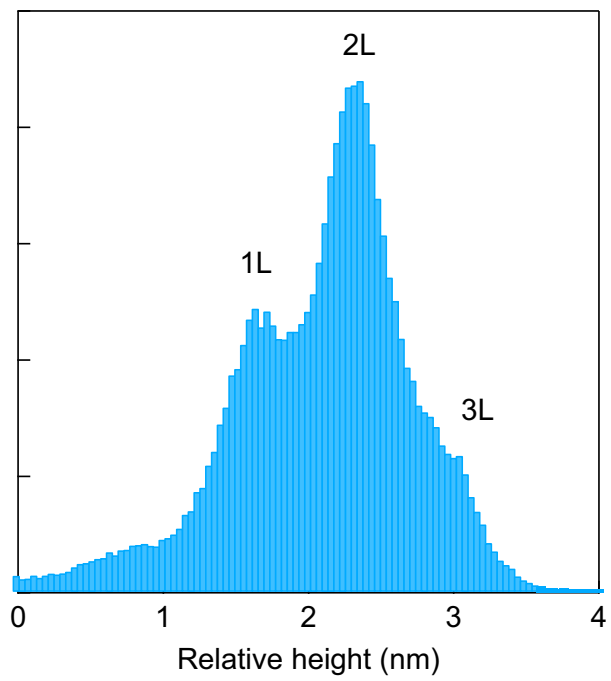

Fig. 1 Structure of atomically thin GaSe. a Schematic drawing of the structure of $\varepsilon$-GaSe. b Simplified schematic drawing of the band structure of monolayer GaSe showing the unusual valence band dispersion. Only the top of the valence and bottom of the conduction band are shown. c AFM image of ultrathin GaSe grown on the surface of GaAs using MBE. The scale bar is $500 \mathrm{~nm}$ long. Labels $1 \mathrm{~L}, 2 \mathrm{~L}$, and $3 \mathrm{~L}$ refer to monolayer, bilayer, and trilayer regions of the film. d Height histogram based on the AFM image in (c)

\section{RESULTS AND DISCUSSION}

\section{Growth of atomically thin GaSe films}

Atomically thin GaSe films were grown on GaAs(111)B substrates by MBE. Supplementary Figures $1 \mathrm{a}$ and $1 \mathrm{~b}$ show the in situ RHEED patterns along two different azimuths of $\mathrm{GaAs}$ during growth, with more details on in-situ growth monitoring available in Supplementary Note 1. We first removed native oxides from the GaAs surface using atomic hydrogen at $300^{\circ} \mathrm{C}$, resulting in a smooth epi-ready surface without further surface roughening which was critical for the deposition of high-quality films (See Supplementary Figure 2). ${ }^{22,23}$ In order to optimize the growth conditions, we have introduced a two-step growth approach. ${ }^{19}$ First, a step at a lower growth temperature and with a short growth time was carried out in order to facilitate nucleation on the substrate, leading to a uniform thickness coverage in the second growth phase later on. After the low-temperature growth step, growth at a higher temperature was conducted during the second growth phase in order to increase the mobility of adatoms, boosting the domain size in the lateral direction (see Supplementary Figure 3 and Supplementary Note 2 for more details). In this way, a highly oriented film can be obtained with a uniformly distributed nominal thickness. The high temperature close to the surface desorption regime significantly reduces the growth rate allowing for easier control of the film thickness by the growth time (see Supplementary Note 2). The morphology examined by atomic force microscopy (AFM) shows sub- $\mu \mathrm{m}$-scale triangular grains of GaSe with well-aligned edges, implying a high degree of order in lattice orientation and demonstrating the high quality of as-grown films (Fig. 1c). By further examining the thickness variance in the histogram, we can determine the interlayer spacing $c / 2$ of $\sim 0.8$ $\mathrm{nm}$. We estimate that over $70 \%$ of the surface is covered by a film that is two layers thick (Fig. 1d). Similar results in terms of morphology control can be reproduced using hydrogenpassivated $\mathrm{H}-\mathrm{Si}(111)$ (Supplementary Figure 4 and Supplementary Note 3 ). This shows that the MBE technique is promising for the growth of atomically thin GaSe with controllable thickness and that it can be extended to other growth substrates.

Lattice structure of atomically thin GaSe resolved by STEM We fist examine the morphology of as-grown GaSe films using scanning transmission electron microscopy (STEM). An energydispersive X-ray spectroscopy (EDAX) cross-sectional analysis of $\sim 8$ layer thick GaSe on GaAs(111)B is shown on Fig. 2a, b. Despite the fact that $\mathrm{Ga}$ atoms are present in both $\mathrm{GaSe}$ and $\mathrm{GaAs}$, we can still differentiate between the films by selecting different elements in the EDAX mapping. The results indicate a uniform distribution of $\mathrm{Ga}$ and $\mathrm{Se}$ in the GaSe films. There is no indication of interdiffusion at the interface as seen in the Se and As elemental distribution map (Fig. 2b), consistent with the van der Waals epitaxial growth mechanism. Our results clearly show the sharp interface without indication of inter-diffusion which is in line with the reported in situ XPS experiments ${ }^{16}$ and a recent study of MBEgrown GaSe on graphene. ${ }^{17,19}$ In a high-magnification crosssectional STEM image, Fig. 2c, we have measured an interlayer spacing $\mathrm{c} / 2$ of $\sim 0.8 \mathrm{~nm}$, which is consistent with the height measurements obtained using an AFM. Figure $2 \mathrm{~d}$ shows an STEM image of a suspended multilayer GaSe membrane. The crystalline lattice can be clearly resolved, belonging to the $\varepsilon$-GaSe polytype 

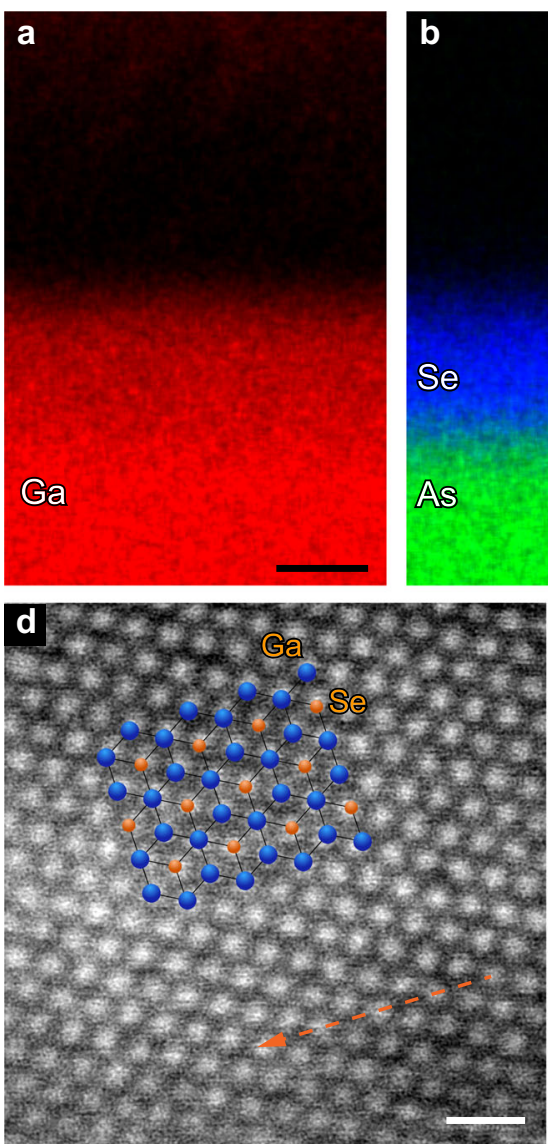

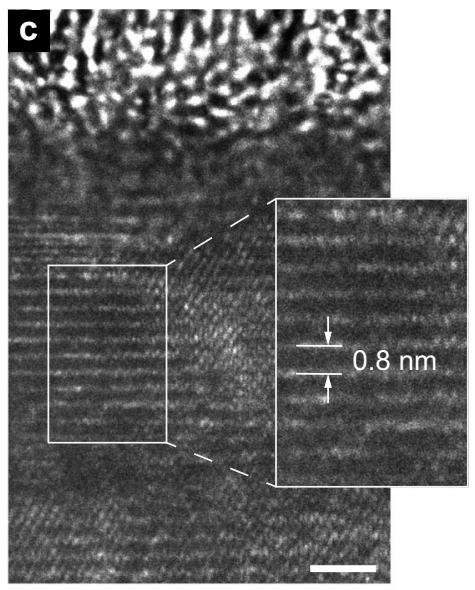

e

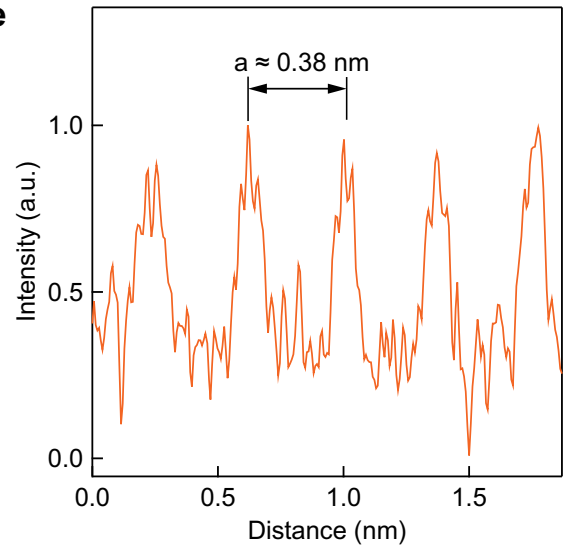

Fig. 2 GaSe film morphology examined using STEM. a EDAX Ga elemental map. b EDAX elemental map of As and Se. c Cross-sectional STEM image of GaSe. Inset shows a zoomed-in region from the outlined rectangle with the extracted out-of-plane lattice constant $c=0.8 \mathrm{~nm}$. Scale bar: $2 \mathrm{~nm}$. d STEM image with the schematic of $\varepsilon$-GaSe lattices with $2 \mathrm{H}(\mathrm{A}+\mathrm{B})$ stacking. Scale bar: $0.5 \mathrm{~nm}$. e Intensity profile along the dashed line in $\mathbf{d}$, showing the in-plane lattice constant $a=0.38 \mathrm{~nm}$

with the $2 \mathrm{H}(\mathrm{A}+\mathrm{B})$ stacking feature, which is also predicted to be the stable polytype in bulk. ${ }^{6,8,24,25}$ The in-plane lattice constant $a$ of GaSe was measured to be $\sim 0.38 \mathrm{~nm}$ based on the intensity profile shown in Fig. 2e. As expected, the modulation of GaSe lattice constants by the substrate cannot be detected, as confirmed by our RHEED patterns.

Electronic properties of as-grown nominal bilayer GaSe

We now turn our attention to the electronic properties of the nominally bilayer GaSe investigated by $k$-PEEM. We first performed the in situ work-function mapping and $\mu \mathrm{m}$-scale XPS measurements to demonstrate the high quality and uniformity of the region of interest (see Supplementary Figure 5 and Supplementary Note 4). Sets of $k$-PEEM images were acquired at different binding energies $E_{\mathrm{B}}$ with respect to the Fermi level $E_{\mathrm{F}}=0 \mathrm{eV}$ in the valence band region of $E_{\mathrm{B}}=2.0-6.7 \mathrm{eV}$ (Fig. 3a), revealing the full valence band structure of atomically thin GaSe films. A clear hexagonal symmetry is shown at different energies highlighting the high degree of crystalline alignment. We can distinguish in the $k$-PEEM images the reciprocal space high-symmetry points $\Gamma, \mathrm{K}$, and $M$. The energy dispersion in reciprocal space along high symmetry directions $\Gamma-\mathrm{K}$ and $\mathrm{\Gamma}-\mathrm{M}$ can then be obtained and is shown in Fig. 3b. We plot the second-derivative of the energy dispersion in Fig. $3 c$ in order to enhance the contrast for better visibility. The VBM globally located at the $\Gamma$ point can be observed to be $0.88 \pm 0.06 \mathrm{eV}$ below the Fermi level $E_{\mathrm{F}}$, demonstrating that the as-grown GaSe film is unintentionally $p$-doped, assuming the band gap of $\sim 2.4 \mathrm{eV}$ for bilayer GaSe. ${ }^{26}$ In bulk GaSe, natural defects such as vacancies, stacking faults and unavoidable impurities such as $\mathrm{Si}$ are known to result in $p$-type doping, ${ }^{27}$ leading to the $E_{\mathrm{F}}-E_{\mathrm{VBM}}$ value of 0.5 to $0.3 \mathrm{eV}{ }^{28}$ where $E_{\mathrm{VBM}}$ is the energy of the VBM. This indicates that our MBE-grown GaSe is somewhat less $p$-doped than those from earlier studies. This cannot be solely explained by the band-alignment with the $p$ doped GaAs substrate (see Supplementary Note 4). Therefore, we speculate that our MBE-grown film contains a lower concentration of vacancies or impurities associated with $p$-type doping than the bulk counterparts or $n$-type GaSe grown on epitaxial graphene. ${ }^{20}$

In order to have a finer look at the upper valence band features of bilayer GaSe, we performed k-PEEM measurements at a higher analyzer energy resolution of $\sim 100 \mathrm{meV}$ and focused on the topmost upper valence band $\left(\Gamma_{1}^{+}\right)$near the $\Gamma$ point. ${ }^{12}$ The acquired band structures and the second-derivative band structures are shown in Fig. $4 a$, b, respectively. We have observed another subband emerging underneath the topmost band with an energy difference of $\sim 0.2 \mathrm{eV}$. This feature cannot be explained by the spin-orbit splitting of the topmost band since the predicted value is in the range of only a few meV. ${ }^{12,29}$ It is more likely that the subband is arising from the mixed signals of the $\Gamma_{1}{ }^{+}$band of exposed monolayer GaSe in the examined region, since the energy difference is in line with theoretical calculations. ${ }^{6,12}$ It is worth noting that the second topmost band, $\left(\Gamma_{3}{ }^{-}\right)$lies below the $\Gamma$ ${ }_{1}^{+}$bands with an energy difference of $\sim 1.1 \mathrm{eV}$ at the $\Gamma$ point, a value which is also comparable with the reported calculations for both the monolayer and bilayer cases making further distinction 
a

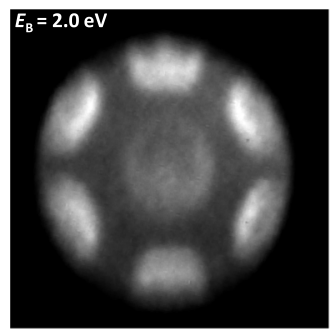

b
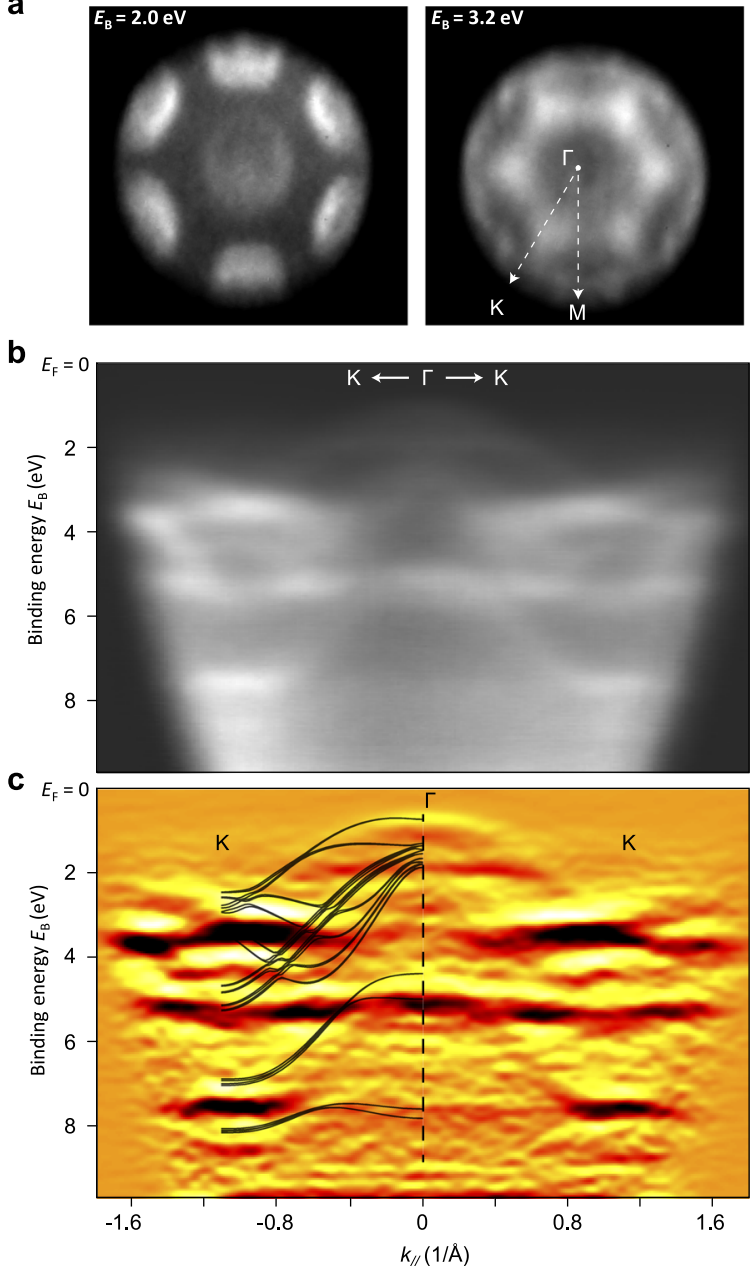
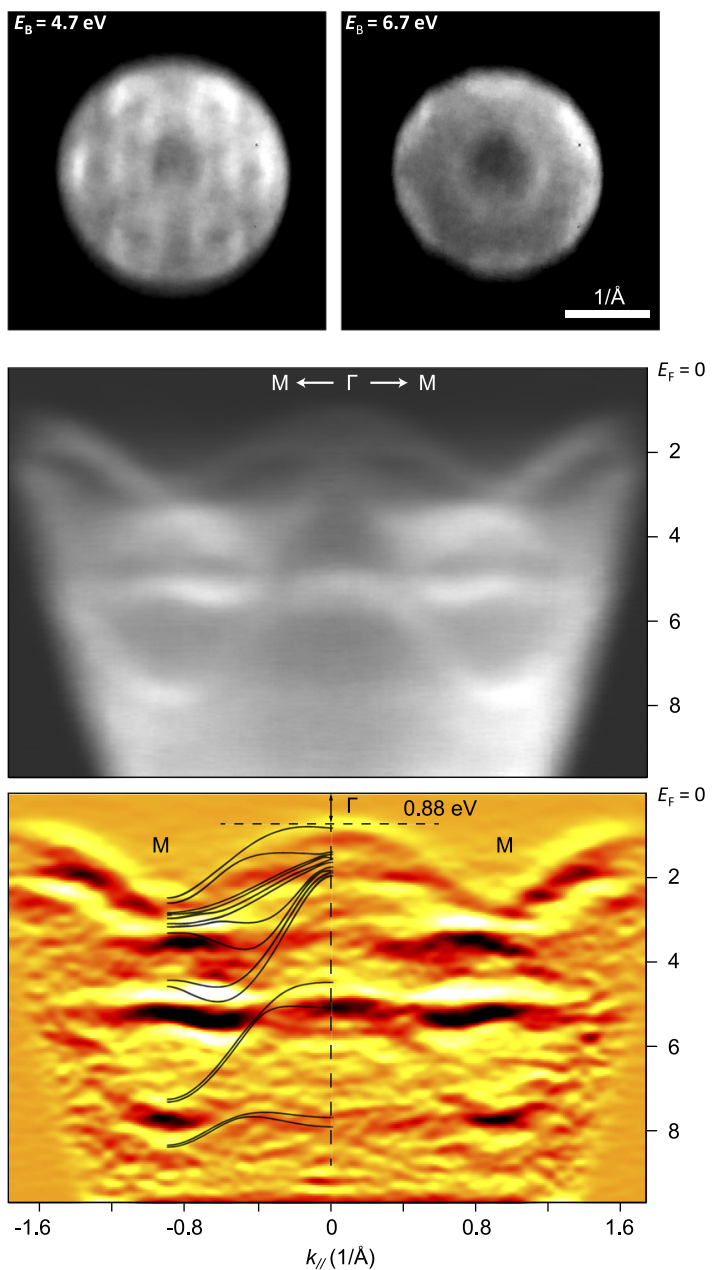

Fig. $3 k$-PEEM images and electronic band-structure of as-grown nominal bilayer GaSe. a $k$-PEEM images acquired at different binding energies $E_{\mathrm{B}}$ (with respect to Fermi level $E_{\mathrm{F}}=0 \mathrm{eV}$ ) from the near-Fermi-level energy towards deep valence-band. $\mathbf{b}$ Electronic band structures along high-symmetry $\Gamma$ to $\mathrm{K}$ (left) and $\Gamma$ to $\mathrm{M}$ (right) directions. c Second-derivative electronic structures with enhanced contrast. The dashed line represents the VBM, which is $\sim 0.88 \mathrm{eV}$ below the Fermi level $E_{\mathrm{F}}$. The calculated band structures are presented as black curves ${ }^{12}$
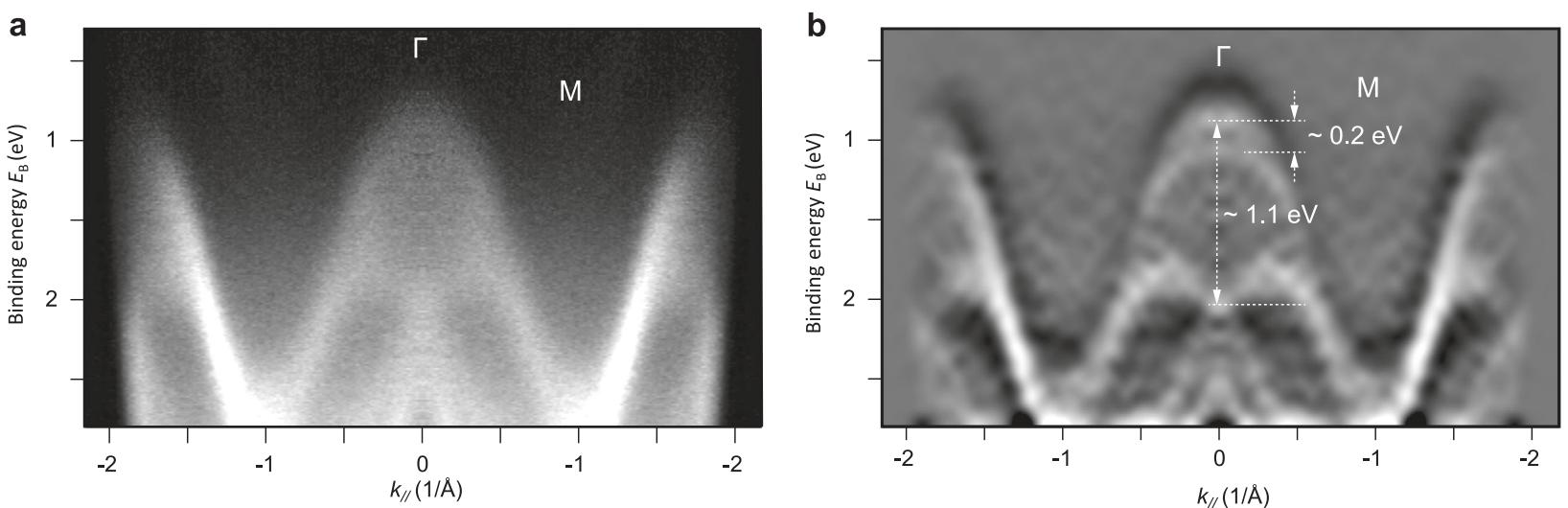

Fig. 4 High-resolution electronic band-structure near the $\Gamma$ point of nominal bilayer GaSe. a Electronic structure near the $\Gamma$ point (b) secondderivative electronic structure from (a). Binding energy $E_{\mathrm{B}}$ is with respect to the Fermi level $E_{\mathrm{F}}=0 \mathrm{eV}$

difficult. $^{6,30}$ While the topmost valence band is the result of hybridization mainly arising from the Se $p_{z}$ orbital, the shapes and the spin splitting in the upper valence bands are the result of mixed $p_{x}, p_{y}$ and $s$ orbital hybridizations. ${ }^{9,29,31}$ The spin-orbit interaction of the valence bands in bilayer GaSe is expected to impart unique optical properties such as 2D spin-dependent excitation and decay by emission of linearly polarized light. ${ }^{31}$

The $k$-PEEM image at the VBM illustrates the constant-energy cross-section of the inverted band structure surrounding the $\Gamma$ point, Fig. 5a. Assuming an isotropic band dispersion near the 


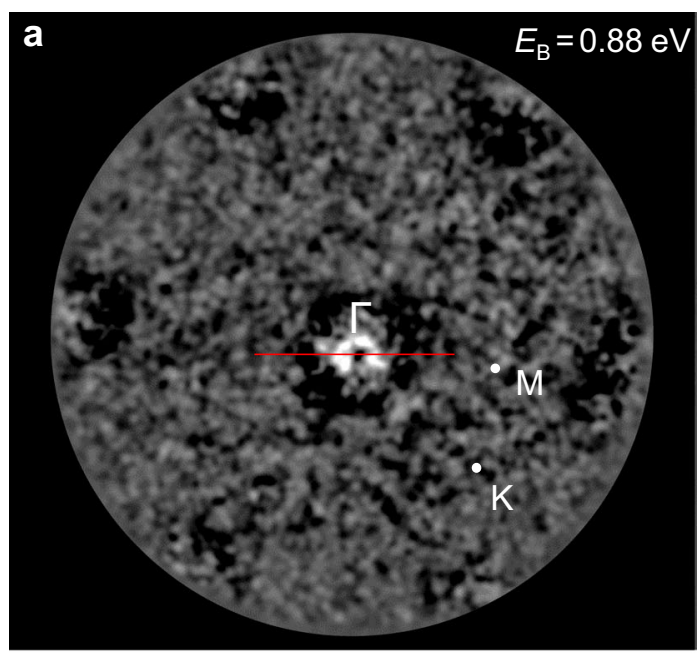

b
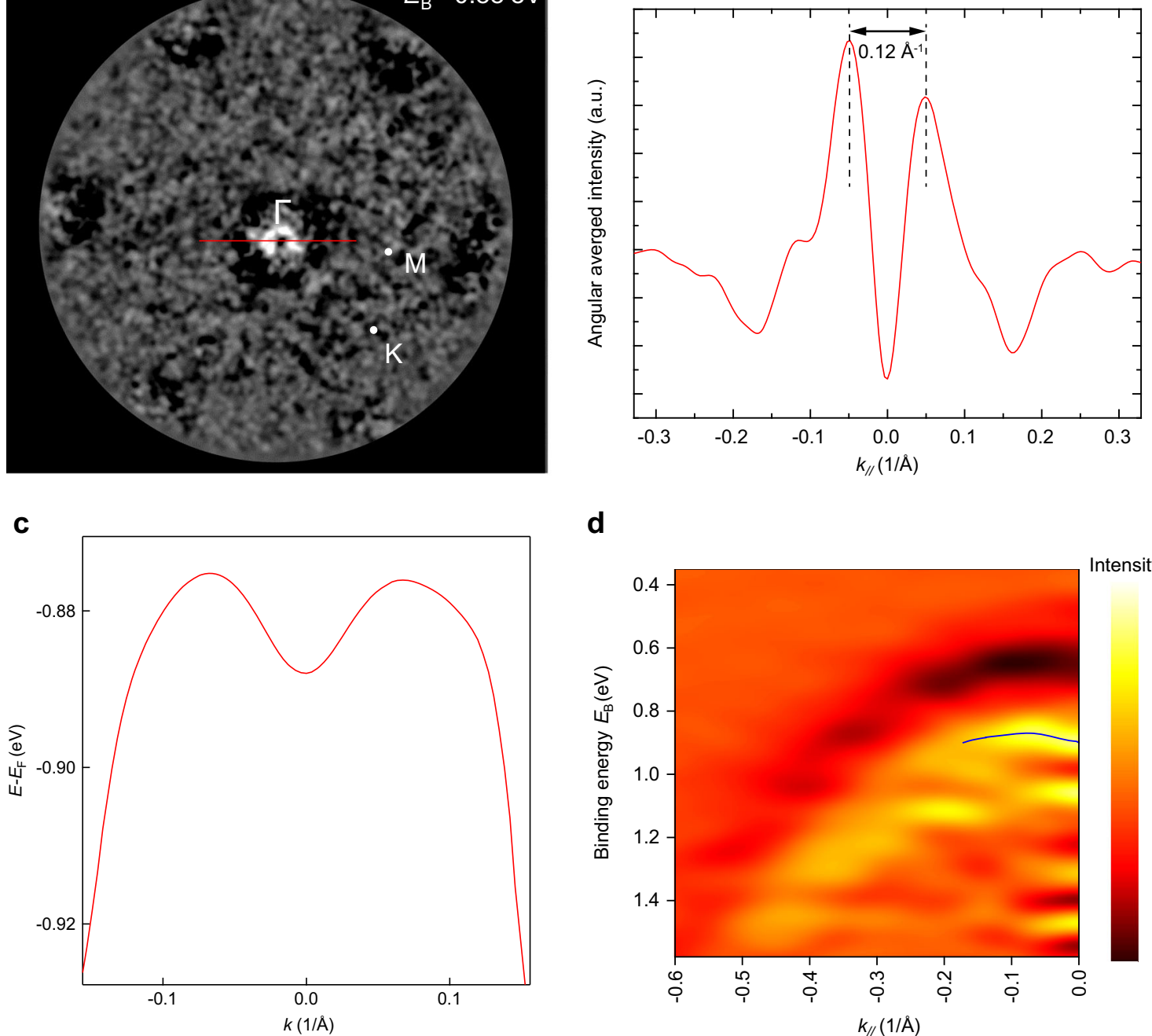

d

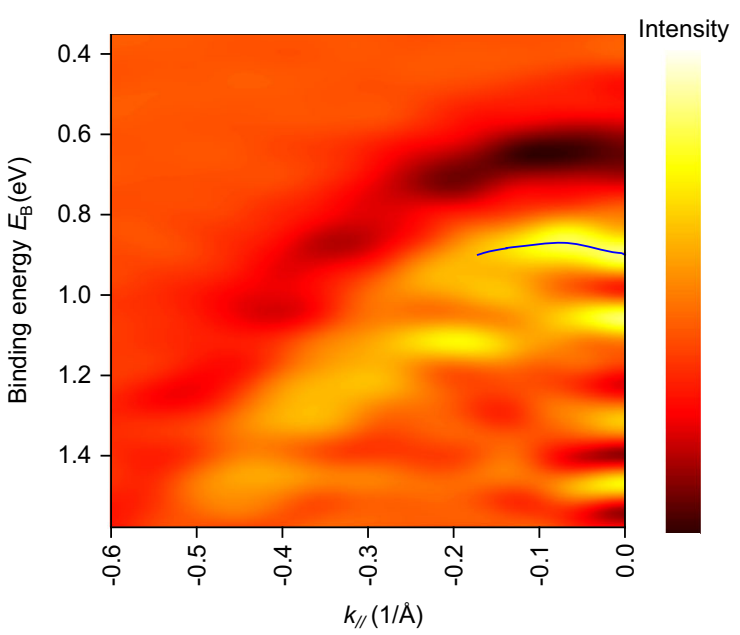

Fig. 5 Inverted band structure of the top-most valence band. a 2nd derivative-filtered $k$-PEEM image at VBM $\left(E_{\mathrm{B}}=0.88 \mathrm{eV}\right)$. The dotted line indicates the basis for the intensity profile analysis around the $\Gamma$ point in (b). $\mathbf{b}$ Angular averaged intensity profile around the $\Gamma$ point $k=0 \AA^{-1}$ through the dotted line in (a). c Dispersion at the top of the valence band reconstructed along the three equivalent $M-\Gamma-M^{\prime}$ directions. $\mathbf{d}$ Angular average $k$-PEEM intensity map around the $\Gamma$ point of the topmost valence band. The solid line indicates the trace of intensity maxima of the topmost valence band structure using energy-distribution curve analysis

VBM, angular averaged intensity profile around the $\Gamma$ point shows a valley with a dip at $\Gamma$ with a diameter of $\sim 0.12 \AA^{-1}$, calculated based on the average separation of the two peaks, Fig. 5b. Here, we show the dispersion relationship for the top of the valence band centered on the $\Gamma$ point obtained by averaging $E_{\mathrm{B}}$ vs. $k$ slices of the $k$-PEEM image stack along the three equivalent $M-\Gamma-M^{\prime}$ directions and fitting the resulting constant $-k$ intensity curves with a Gaussian function.

The topmost valence band traced with the intensity maximum curves clearly shows the VBM located away from the $\Gamma$ point (Fig. 5c). The multi-peak analysis of the energy dispersion curves of the upper valence band near the $\Gamma$ point by Gaussian peak fitting allows a systematic tracing of the dispersion relationship. It reveals the non-parabolic, inverted band structure (solid curve in Fig. 5d), as predicted for monolayer to few-layer thick GaSe. We estimate the depth of the valley at $\Gamma$ to be $0.012 \mathrm{eV}$ and the radius of the inverted structure in the $k$-space, $k_{0}$ ( $\Gamma$ to the VBM) to be $0.068 \AA^{-1}$. Due to the unique band structure, the effective mass can vary dramatically over a short range of $k$-space (see Supplementary Figure 6 and Supplementary Note 5). After polynomial approximation of the dispersion curve in the range of the inverted region in the $k$-space, the $k$-dependent effective mass can be calculated from the inverse of the curvature $\left(1 / m^{*}=1 / \hbar^{2} \times d^{2} E / d k^{2}\right)$ as shown in Supplementary Figure 6 . This calculation gives the positive hole effective mass value near $\Gamma$ that rapidly increases until the Van Hove singularity point at $\sim 0.03 \AA^{-1}$ where it changes its sign as predicted. The hole effective mass value of $\sim 0.6 \mathrm{~m}_{\mathrm{o}}$ at $\Gamma$ lies in a reasonable range considering predicted effective mass values of $0.54 \mathrm{~m}_{\mathrm{o}}$ and $0.9 \mathrm{~m}_{\mathrm{o}}$, respectively for monolayer and bilayer $\beta$-GaSe. ${ }^{9}$ We thus expect that the nominal bilayer GaSe presented here can serve as an ideal material platform for further explorations of novel electronic properties arising from its inverted band structure.

In conclusion, we have used MBE to grow atomically thin GaSe films. The high quality of the films at micro to nanoscale was revealed by different characterization techniques including STEM, PEEM, and AFM. We report the measurement of the electronic band structure of nominal bilayer GaSe films obtained using $k$ PEEM. At the atomically thin limit, GaSe exhibits an inverted band dispersion at the $\Gamma$ point, leading to the shift of the VBM away from the $\Gamma$ point. The results shed light not only on the growth of 
monochalcogenides but also on new potential applications based on their unusual valence band dispersion.

\section{METHODS}

MBE setup and material growth

The growth was carried out in an Omicron MBE with $\sim 10^{-10}$ mbar base pressure. Cleaved $1 \times 1 \mathrm{~cm}^{2} \mathrm{GaAs}(111) \mathrm{B}$ substrates were outgassed up to $500{ }^{\circ} \mathrm{C}$ for at least $30 \mathrm{~min}$ prior to growth, and the native oxides were removed by atomic $\mathrm{H}$ source at $300^{\circ} \mathrm{C}$ for $30 \mathrm{~min}$. Hydrogen molecules were dissociated by a tungsten filament with Joule heating at $70 \mathrm{~W}$ and were introduced into the chamber via a leak valve with a pressure of $3 \times$ $10^{-7} \mathrm{mbar}$. The growth was first conducted at $450^{\circ} \mathrm{C}$ for $5 \mathrm{~min}$ and paused by closing the mechanical shutters. The temperature was then ramped up to $530^{\circ} \mathrm{C}$ with the ramping rate of $10^{\circ} \mathrm{C} / \mathrm{min}$ and the growth resumed for another $30 \mathrm{~min}$ by opening the shutters. The temperature above $400^{\circ} \mathrm{C}$ was monitored using a pyrometer during the entire growth process. The Ga cell was operated at $910^{\circ} \mathrm{C}$ with a Se/Ga ratio $\sim 12$ calibrated using a quartz crystal microbalance. A RHEED camera (STAIB Co.) was used to monitor the growth in-situ. Nominal bilayer GaSe samples were grown at the optimized conditions at least six times to check the reproducibility and material characterization. As-grown films were imaged and their uniformity checked using an AFM (Asylum Research Cypher) operating in ac mode.

\section{Material transfer and STEM sample preparation}

The cross-sectional lamella for STEM was prepared by standard focused ion beam. A thick amorphous carbon layer was deposited on the as-grown samples to protect the films before the focused ion beam cross-sectioning.

For the preparation of suspended GaSe films on TEM grids, we introduced the electrochemical delamination method. ${ }^{32,33}$ The as-grown films were first coated with PMMA. Direct current voltage of $-5 \mathrm{~V}$ was applied to the PMMA/GaSe/GaAs acting as the cathode thanks to the high conductivity of heavily $p^{++}$-doped-GaAs substrates. A platinum wire was used as the anode in an aqueous solution of $\mathrm{K}_{2} \mathrm{~S}_{2} \mathrm{O}_{8}(0.05 \mathrm{mM})$ used as the electrolyte. $\mathrm{H}_{2}$ bubbles formed at the $\mathrm{GaSe} / \mathrm{GaAs}$ interface and the delamination took place. After transferring the floating film onto the TEM grid, PMMA was dissolved in acetone.

\section{STEM microscopy and analysis}

The STEM experiments were performed using a FEI Titan Themis 300 double Cs corrected microscope at a $80 \mathrm{kV}$ acceleration voltage to avoid specimen damage induced by the beam. The scanning probe had a 28 mrad semiconvergence angle which allows achieving a resolution close to $1 \AA$ A. The data was acquired under annular dark field conditions using an annular detector with a semicollection angle between 40 and 200 mrad.

\section{Photoemission electron momentum microscopy}

In order to avoid charging effects during the PEEM experiment, we used Zn-doped GaAs(111)B (Semiconductor Wafer Inc.) with doping concentration of $2 \times 10^{18}-5 \times 10^{19} \mathrm{~cm}^{-3}$ as a substrate. The $\mu \mathrm{m}$-scale PEEM, $k$-PEEM, and XPS measurements of GaSe were performed on the same area with a NanoESCA Mkl spectro-microscope (Scienta Omicron) in ultra-high vacuum $\left(2 \times 10^{-10} \mathrm{mbar}\right)$ and room temperature conditions. ${ }^{34,35}$ For threshold secondary electron PEEM imaging and $k$-PEEM band structure imaging, the ultraviolet (UV) illumination was provided by a vacuum UV helium cold cathode lamp ( $h v=21.2 \mathrm{eV})$ at an off-normal angle of $65^{\circ}$. The excitation photon energy of $21.2 \mathrm{eV}$ ensures efficient photoemission of valence band electrons due to the high cross-section values for the main valence band states of Ga $4 p, \mathrm{Ga} 4 s$, Se $4 p$, and Se $4 s$ at the photon energy of the helium lamp.

For the same area $k$-PEEM and XPS measurements, a field aperture located at the first intermediate image plane in the PEEM column was closed in order to select a region of $\sim 20 \mu \mathrm{m}$ in diameter for simultaneous $\mu \mathrm{m}$-scale electronic band structure imaging and chemical analysis. For $k$ PEEM, pass energies of 50 and $25 \mathrm{eV}$ with an entrance slit size of $1 \mathrm{~mm}$ were used resulting in analyzer resolution of 200 and $100 \mathrm{meV}$, respectively. For the core-level XPS analysis, pass energy of $50 \mathrm{eV}$ for entrance slit size of $2 \mathrm{~mm}$ was used providing an analyzer resolution of $400 \mathrm{meV}$.

Work-function mapping was performed by fitting the onset of the secondary electron emission signal in the spectrum at each image pixel of the PEEM image series, thereby providing a local value of the work function. The values were shifted by $0.98 \mathrm{eV}$ compensating for the Schottky effect. ${ }^{36}$ The dispersion relationship for the top valence band structure centered on the $\Gamma$ point $\left(k_{/ /}=0 \AA^{-1}\right)$ was obtained by first averaging the $E_{\mathrm{bin}}$ vs. $k$ slices of the $k$-PEEM image stack along the three equivalent $\mathrm{MGM}^{\prime}$ directions. Then, each constant- $k$ intensity curve was fitted with a Gaussian peak yielding a peak position which was used to trace the dispersion relationship in the range of the inverted structure $\left(E_{\mathrm{B}}\right.$ $\left.=0.86-0.91 \mathrm{eV} ; k_{/ /}=0-0.15 \AA^{-1}\right)$.

\section{Data availability}

The data that support the findings of this study are available from the corresponding author on reasonable request.

\section{ACKNOWLEDGEMENTS}

We thank M. Cantoni and D. Alexander (CIME) for FIB operation and for support with electron microscopy. We thank P. Mettraux for XPS setup and experiments. The momentum microscopy was performed in the Nanocharacterization Platform (PFNC) of MINATEC Campus within CEA-Grenoble research center. This project has received funding from the EU-H2020 research and innovation program under grant agreement No 654360 NFFA-Europe. We thank N. Gambacorti for coordinating the access under the NFFA-Europe scheme. This work was financially supported by the European Research Council (grants no. 240076 and 682332) and has received funding from the European Union's Horizon 2020 research and innovation programme under grant agreement No. 696656 (Graphene Flagship) and European Union's Seventh Framework Programme FP7/2007-2013 under Grant Agreement No. 318804 (SNM).

\section{AUTHOR CONTRIBUTIONS}

M.W.C. performed MBE growth, H.K. performed PEEM, $k$-PEEM, and XPS analysis, D.O. performed TEM analysis. A. Kuc performed theoretical calculations which were supervised by T.H. O.R. worked on PEEM and k-PEEM measurements. A.K. supervised the project. M.W.C., H.K. and A.K. wrote the manuscript. All authors discussed the results.

\section{ADDITIONAL INFORMATION}

Supplementary information accompanies the paper on the npj 2D Materials and Applications website (https://doi.org/10.1038/s41699-017-0047-x).

Competing interests: The authors declare no competing financial interests.

Publisher's note: Springer Nature remains neutral with regard to jurisdictional claims in published maps and institutional affiliations.

\section{REFERENCES}

1. Radisavljevic, B., Radenovic, A., Brivio, J., Giacometti, V. \& Kis, A. Single-layer $\mathrm{MoS}_{2}$ transistors. Nat. Nanotechnol. 6, 147-150 (2011).

2. Wang, Q. H., Kalantar-Zadeh, K., Kis, A., Coleman, J. N. \& Strano, M. S. Electronics and optoelectronics of two-dimensional transition metal dichalcogenides. Nat. Nanotechnol. 7, 699-712 (2012).

3. Chhowalla, M. et al. The chemistry of two-dimensional layered transition metal dichalcogenide nanosheets. Nat. Chem. 5, 263-275 (2013).

4. Zhou, X. et al. Strong second-harmonic generation in atomic layered GaSe. J. Am. Chem. Soc. 137, 7994-7997 (2015).

5. Zhou, Y. et al. Epitaxy and photoresponse of two-dimensional GaSe crystals on flexible transparent mica sheets. ACS Nano 8, 1485-1490 (2014).

6. Ma, Y., Dai, Y., Guo, M., Yu, L. \& Huang, B. Tunable electronic and dielectric behavior of GaS and GaSe monolayers. Phys. Chem. Chem. Phys. 15, 7098-7105 (2013).

7. Cao, T., Li, Z. \& Louie, S. G. Tunable magnetism and half-metallicity in hole-doped monolayer GaSe. Phys. Rev. Lett. 114, 236602 (2015).

8. Li, X. et al. Controlled vapor phase growth of single crystalline, two-dimensional GaSecrystals with high photoresponse. Sci. Rep. 4, 5497 (2014).

9. Rybkovskiy, D. V., Osadchy, A. V. \& Obraztsova, E. D. Transition from parabolic to ring-shaped valence band maximum in few-layer GaS, GaSe, and InSe. Phys. Rev. B 90, 235302 (2014).

10. Wu, S. et al. Magnetisms in p-type monolayer gallium chalcogenides (GaSe, GaS). arXiv:1409.4733 [cond-mat] (2014).

11. Wickramaratne, D., Zahid, F. \& Lake, R. K. Electronic and thermoelectric properties of van der Waals materials with ring-shaped valence bands. J. Appl. Phys. 118, 075101 (2015). 
12. Kuc, A. et al. High-performance 2D p-type transistors based on GaSe Layers: an Ab Initio study. Adv. Electron. Mater. 3, 1600399 (2017).

13. Vinh, L. T., Eddrief, M., Sébenne, C., Sacuto, A. \& Balkanski, M. Heteroepitaxy of GaSe layered semiconductor compound on $\mathrm{Si}(111) 7 \times 7$ substrate: a Van der Waals epitaxy? J. Cryst. Growth 135, 1-10 (1994).

14. Ueno, K., Abe, H., Saiki, K. \& Koma, A. Heteroepitaxy of layered semiconductor GaSe on a GaAs(111)B surface. Jpn J. Appl. Phys. 30, L1352 (1991).

15. Ueno, K., Takeda, N., Sasaki, K. \& Koma, A. Investigation of the growth mechanism of layered semiconductor GaSe. Appl. Surf. Sci. 113-114, 38-42 (1997).

16. Zheng, Y. et al. GaSeSi(111) heteroepitaxy: the early stages of growth. J. Cryst. Growth 162, 135-141 (1996).

17. Ben Aziza, Z. et al. van der Waals Epitaxy of GaSe/graphene heterostructure: electronic and interfacial properties. ACS Nano 10, 9679-9686 (2016).

18. Palmer, J. E., Saitoh, T., Yodo, T. \& Tamura, M. Growth of GaSe on As-passivated Si (111) substrates. J. Cryst. Growth 147, 283-291 (1995).

19. Rumaner, L. E., Gray, J. L. \& Ohuchi, F. S. Nucleation and growth of GaSe on GaAs by Van der Waal epitaxy. J. Cryst. Growth 177, 17-27 (1997).

20. Ben Aziza, Z. et al. Tunable quasiparticle band gap in few-layer GaSe/graphene van der Waals heterostructures. Phys. Rev. B 96, 035407 (2017).

21. Yuan, X. et al. Arrayed van der Waals vertical heterostructures based on $2 D$ GaSe grown by molecular beam epitaxy. Nano Lett. 15, 3571-3577 (2015).

22. Khatiri, A., Krzyzewski, T. J., McConville, C. F. \& Jones, T. S. Atomic hydrogen cleaning of low-index GaAs surfaces. J. Cryst. Growth 282, 1-6 (2005).

23. Sugaya, T. \& Kawabe, M. Low-temperature cleaning of GaAs substrate by atomic hydrogen irradiation. Jpn J. Appl. Phys. 30, L402 (1991).

24. Zhu, Z., Cheng, Y. \& Schwingenschlögl, U. Topological phase transition in layered GaS and GaSe. Phys. Rev. Lett. 108, 266805 (2012).

25. Li, X. et al. Revealing the preferred interlayer orientations and stackings of twodimensional bilayer gallium selenide crystals. Angew. Chem. Int. Ed. 54, 2712-2717 (2015).

26. Jung, C. S. et al. Red-to-ultraviolet emission tuning of two-dimensional gallium sulfide/selenide. ACS Nano 9, 9585-9593 (2015).

27. Brudnyi, V. N., Sarkisov, S. Y. \& Kosobutsky, A. V. Electronic properties of GaSe, InSe, GaS and GaTe layered semiconductors: charge neutrality level and interface barrier heights. Semicond. Sci. Technol. 30, 115019 (2015).

28. Lang, O., Tomm, Y., Schlaf, R., Pettenkofer, C. \& Jaegermann, W. Single crystalline $\mathrm{GaSe} / \mathrm{WSe}_{2}$ heterointerfaces grown by van der Waals epitaxy. II. Junction characterization. J. Appl. Phys. 75, 7814 (1994).
Large-grain MBE-grown GaSe on GaAs with a Mexican hat-like valence $M-W$ Chen et al.

29. Do, D. T., Mahanti, S. D. \& Lai, C. W. Spin splitting in 2D monochalcogenide semiconductors. Sci. Rep. 5, 17044 (2015).

30. Rybkovskiy, D. V. et al. Size-induced effects in gallium selenide electronic structure: the influence of interlayer interactions. Phys. Rev. B 84, 085314 (2011).

31. Li, P. \& Appelbaum, I. Symmetry, distorted band structure, and spin-orbit coupling of group-III metal-monochalcogenide monolayers. Phys. Rev. B 92, 195129 (2015).

32. Wang, Y. et al. Electrochemical delamination of CVD-grown graphene film: toward the recyclable use of copper catalyst. ACS Nano 5, 9927-9933 (2011).

33. Koefoed, L. et al. Facile electrochemical transfer of large-area single crystal epitaxial graphene from $\operatorname{lr}\left(\begin{array}{lll}1 & 1 & 1\end{array}\right)$. J. Phys. D. Appl. Phys. 48, 115306 (2015).

34. Escher, M. et al. NanoESCA: a novel energy filter for imaging $x$-ray photoemission spectroscopy. J. Phys. Condens. Matter 17, S1329 (2005).

35. Escher, M., Winkler, K., Renault, O. \& Barrett, N. Applications of high lateral and energy resolution imaging XPS with a double hemispherical analyser based spectromicroscope. J. Electron Spectrosc. Relat. Phenom. 178-179, 303-316 (2010).

36. Zagonel, L. F. et al. Orientation-dependent work function of in situ annealed strontium titanate. J. Phys. Condens. Matter 21, 314013 (2009).

(i) Open Access This article is licensed under a Creative Commons Attribution 4.0 International License, which permits use, sharing, adaptation, distribution and reproduction in any medium or format, as long as you give appropriate credit to the original author(s) and the source, provide a link to the Creative Commons license, and indicate if changes were made. The images or other third party material in this article are included in the article's Creative Commons license, unless indicated otherwise in a credit line to the material. If material is not included in the article's Creative Commons license and your intended use is not permitted by statutory regulation or exceeds the permitted use, you will need to obtain permission directly from the copyright holder. To view a copy of this license, visit http://creativecommons. org/licenses/by/4.0/.

(C) The Author(s) 2018 